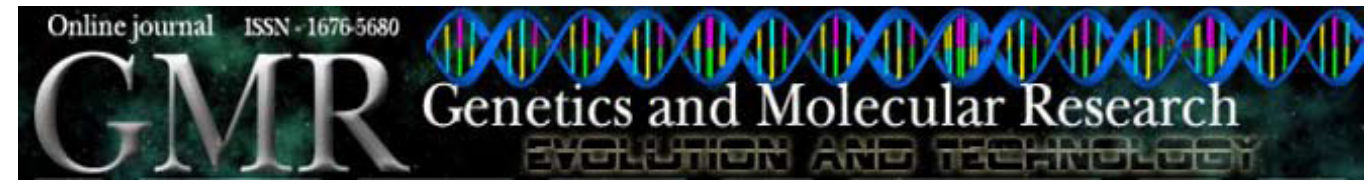

\title{
Transferability of microsatellite markers among economically and ecologically important galliform birds
}

\author{
N. Bech ${ }^{1}$, C. Novoa ${ }^{2}$ J.F. Allienne ${ }^{1}$ and J. Boissier ${ }^{1}$ \\ ${ }^{1}$ Laboratoire de Biologie et d'Écologie Tropicale et Méditerranéenne, \\ Centre National de la Recherche Scientifique, \\ Université de Perpignan, Perpignan Cedex, France \\ ${ }^{2}$ Office National de la Chasse et de la Faune Sauvage, \\ Direction des Etudes et de la Recherche, Prades, France \\ Corresponding author: N. Bech \\ E-mail: nicolas.bech@univ-perp.fr
}

Genet. Mol. Res. 9 (2): 1121-1129 (2010)

Received January 8, 2010

Accepted March 20, 2010

Published June 15, 2010

DOI $10.4238 /$ vol9-2gmr760

\begin{abstract}
We used the partially sequenced genomes of the turkey and chicken to find a large number of microsatellite markers. We then characterized 10 polymorphic microsatellite markers developed by cross-species amplification from economically and ecologically important birds to various European subspecies of the grey partridge. Even though we used cross-species amplification, a high degree of polymorphism was conserved in all microsatellite markers. Cross-species amplification from
\end{abstract}


birds of economic and ecological interest, such as chicken and turkey, could be an attractive approach to develop microsatellite markers and to use these to manage wild and captive populations of other galliforms, such as the grey partridge.

Key words: Grey partridge; Turkey; Chicken; Microsatellite markers; Cross-species amplification

\section{INTRODUCTION}

Among galliforms, the chicken (Gallus gallus) and turkey (Meleagris gallopa$v o$ ) are of particular economic interest, and their genomes are respectively entirely and partially sequenced. Several hundred primer pairs have already been defined to amplify microsatellite markers in these two species. In this study, we proposed to test the transferability of these markers to the grey partridge (Perdix perdix), another galliform species of ecological and economic interest. Considering that the galliform phylogenetic tree shows that the chicken is basal and that the turkey is a sister taxon of the grey partridge (Dimcheff et al., 2002; Kimball et al., 1999), we hypothesized that primer pairs identified in chicken and conserved in turkey (or conversely) should also be conserved in grey partridge. The grey partridge is represented by 8 allopatric sub-species occurring in Eurasia. Like other galliforms, such as Capercaillie (Segelbacher and Storch, 2002) or rock ptarmigan (Bech et al., 2009), global warming would cause the loss of suitable habitat and would thus increase population fragmentation of the grey partridge. In France, this habitat fragmentation would lead to the geographical isolation of two sub-species, Perdix perdix hispaniensis (included in the red data book of threatened species) in the Pyrenees and Perdix perdix armoricana in the north of France. Moreover, P. p. hispaniensis and $P$. p. armoricana represent important small game species, and because they are depleted by hunting, they are regularly restocked. Because restocking involves individuals originating from distinct European sub-species, wild populations display a risk of genetic introgression (Martin et al., 2003).

Microsatellite markers are a very useful tool for both ecological monitoring of wild populations and stock management and enhancement of captive-bred individuals. In this paper, we present the characterization of microsatellite markers usable for the European grey partridge.

\section{MATERIAL AND METHODS}

\section{Cross-species amplification}

In this study, we tested 138 microsatellite primer pairs (see Supporting Information Online; Supplementary Material) on the grey partridge, which have been successfully amplified in both chicken and turkey. Three other criteria were also considered: i) primer pairs would show high annealing temperature in source species; ii) the microsatellite loci would be polymorphic in their source species, and iii) the microsatellite loci would be located on different chicken chromosomes in order to avoid potential linkage disequilibrium. Sampling included liver and feather samples, 
which were collected during the hunting season $(\mathrm{N}=82)$. Main samples came from two distinct wild sub-species ( $P . p$. hispaniensis and $P . p$. armoricana). Some other samples came from stockbreeding with an imprecise geographical origin. Genomic DNA was extracted using silica columns (e.Z.N.A from OMEGA BIO-TEK), according to the manufacturer protocol. Polymerase chain reaction was performed using the QIAGEN multiplex kit. Multiplexes were carried out according to the manufacturer standard microsatellite amplification protocol in a final volume of $10 \mu \mathrm{L}$ and at $57^{\circ} \mathrm{C}$ for annealing temperature.

\section{Statistical analysis}

Departures from Hardy-Weinberg expectations and linkage disequilibria were assessed for each specific microsatellite markers using the exact tests (1000 permutations) implemented in GENEPOP version 3.4 (Raymond and Rousset, 1995). Polymorphism of microsatellite markers, which gave us a specific amplification, was estimated with the number of alleles (A), and expected heterozygosity $\left(\mathrm{H}_{\mathrm{e}}\right.$ ) (Weir and Cockerham, 1984) using FSTAT version 2.9.3.2 (Goudet, 2001) with 800 permutations.

\section{RESULTS}

Among 138 loci tested, 76 gave amplification with only one specific product (see Supporting Information Online; Supplementary Material). Of these, 10 microsatellite markers were polymorphic (Table 1). Among these, no evidence of linkage disequilibrium was detected $(\mathrm{P}<0.001$, significance threshold adjusted with the Bonferroni's procedure, for 45 tests). After Bonferroni's correction for multiple comparison $(\mathrm{P}<0.005)$, only two loci showed significant deviation from HardyWeinberg expectations: MNT412 and MNT45 $\left(\mathrm{F}_{\mathrm{IS}}>0\right)$. This heterozygosity deficit could be interpreted as the result of a Walhund effect because our sampling included several samples from different geographical regions but not real field populations, which are not yet determined. Concerning polymorphism, the number of alleles per locus ranged from 3 to 19 and gene diversity ranged from 0.51 to 0.89 (Table 1).

\section{DISCUSSION}

This study shows that genomic sequence databases of economically important animals are useful tools to identify conserved genetic markers. Because there are not many microsatellites in avian species (Primmer et al., 1997), cross-species amplification from birds of economic interest could be an attractive approach to manage wild and captive populations of other birds such as the grey partridge. In birds, it has been shown that the polymorphism rate of microsatellite markers decreases with the increase in genetic distance from the species from which they have developed (Ellegren et al., 1995; Primmer et al., 1996). With the use of cross-species amplification, a high polymorphism was conserved in our microsatellite markers, which is very promising in terms of usefulness. Studies based on these markers are now underway. 
N. Bech et al..

1124

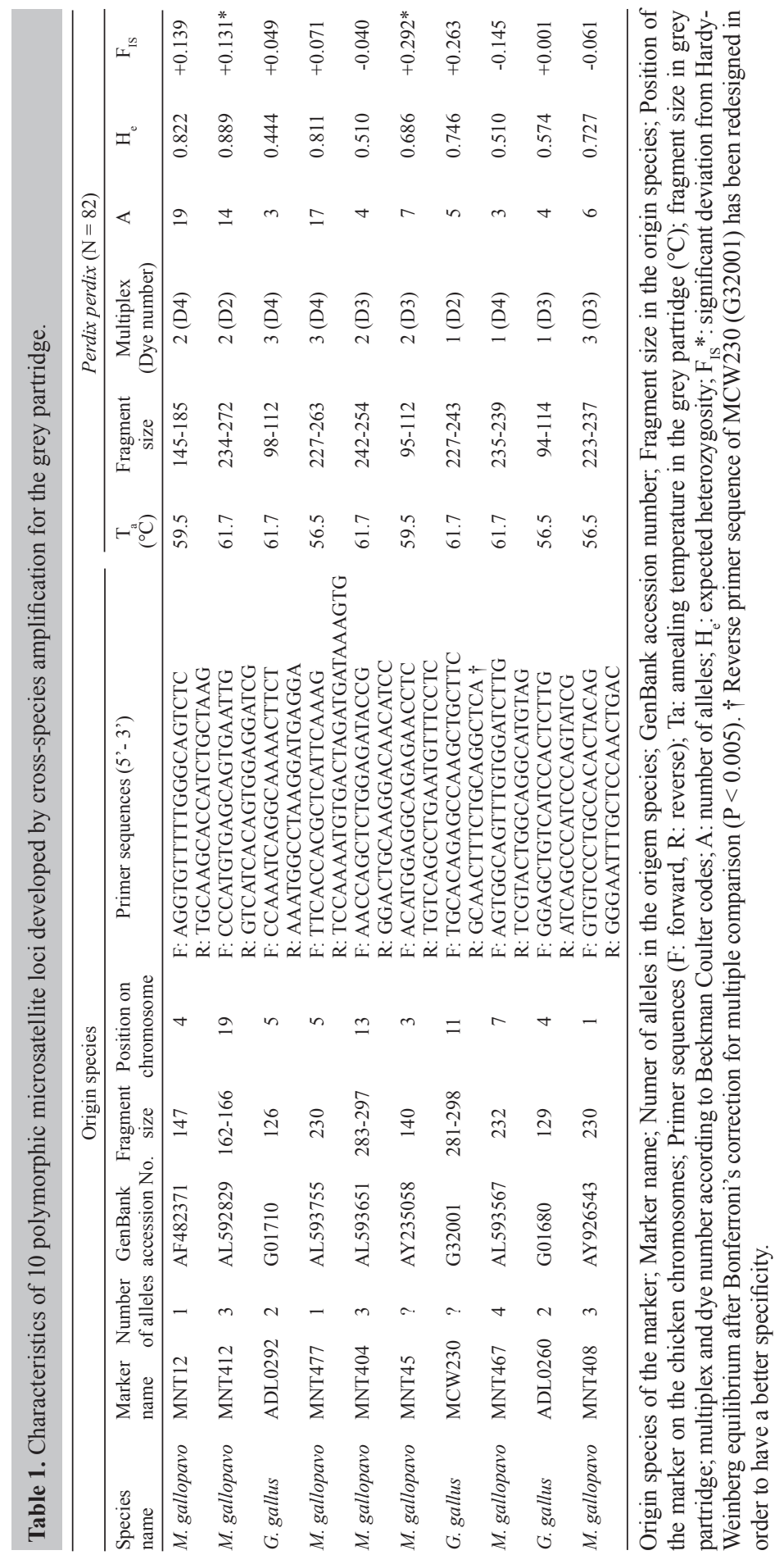

Genetics and Molecular Research 9 (2): 1121-1129 (2010)

CFUNPEC-RP www.funpecrp.com.br 


\section{ACKNOWLEDGMENTS}

Research supported by Bureau des Ressources Génétiques, Office National de la Chasse et de la Faune Sauvage, Ministère de l'Enseignement Supérieur et de la Recherche Scientifique and Centre National de la Recherche Scientifique (all in France). Equally, we would like to thank especially David Ripoll and Mathieu Blanchard who contributed equally to this project.

\section{REFERENCES}

Bech N, Boissier J, Drovetski S and Novoa C (2009). Population genetic structure of rock ptarmigan in the 'sky islands' of French Pyrenees: implications for conservation. Anim. Conserv. 12: 138-146.

Dimcheff DE, Drovetski SV and Mindell DP (2002). Phylogeny of Tetraoninae and other galliform birds using mitochondrial 12S and ND2 genes. Mol. Phylogenet. Evol. 24: 203-215.

Ellegren H, Primmer CR and Sheldon BC (1995). Microsatellite 'evolution': directionality or bias? Nat. Genet. 11: 360362.

Goudet J (2001). FSTAT, a program to estimate and test gene diversities and fixation indices (version 2.9.3). Available at [http://www2.unil.ch/popgen/softwares/fstathtml]. Accessed May 31, 2010.

Kimball RT, Braun EL, Zwartjes PW, Crowe TM, et al. (1999). A molecular phylogeny of the Pheasants and Partridges suggests that these lineages are not monophyletic. Mol. Phylogenet. Evol. 11: 38-54.

Martin JF, Novoa C, Blanc-Manel S and Taberlet P (2003). Les populations de perdrix grise des Pyrénées (Perdix perdix hispaniensis) ont-elles subi une introgression génétique à partir d'individus d'élevage? Analyse du polymorphisme de l'ADN mitochondria. Les Actes du BRG 4: 115-126.

Primmer CR, Moller AP and Ellegren H (1996). A wide-range survey of cross-species microsatellite amplification in birds. Mol. Ecol. 5: 365-378.

Primmer CR, Raudsepp T, Chowdhary BP, Moller AP, et al. (1997). Low frequency of microsatellites in the Avian genome. Genome Res. 7: 471-482.

Raymond M and Rousset F (1995). Genepop (version 1.2): population genetics software for exact tests and ecumenicism. J. Hered. 86: 248-249.

Segelbacher G and Storch I (2002). Capercaillie in the Alps: genetic evidence of metapopulation structure and population decline. Mol. Ecol. 11: 1669-1677.

Weir BS and Cockerham CC (1984). Estimating F-statistics for the analysis of population structure. Evolution 38: 1358-1370 


\section{Supplementary material.}

\begin{tabular}{|c|c|c|c|}
\hline Origin species & Marker name & GenBank accession No. & Reference \\
\hline G. gallus & ADL0019 & L23887 & (Reed et al., 2000a) \\
\hline G. gallus & ADL0039 & L23917 & (Reed et al., 2000a) \\
\hline G. gallus & ADL0044 & L23909 & (Reed et al., 2000a) \\
\hline G. gallus & ADL0102 & G01547 & (Reed et al., 2000a) \\
\hline G. gallus & ADL0106 & G01550 & (Reed et al., 2000a) \\
\hline G. gallus & ADL0114 & G01726 & (Reed et al., 2000a) \\
\hline G. gallus & ADL0127 & G01736 & (Reed et al., 2000a) \\
\hline G. gallus & ADL0132 & G01740 & (Reed et al., 2000a) \\
\hline G. gallus & ADL0136 & G01561 & (Reed et al., 2000a) \\
\hline G. gallus & ADL0138 & G01563 & (Reed et al., 2000a) \\
\hline G. gallus & ADL0143 & G01568 & (Reed et al., 2000a) \\
\hline G. gallus & ADL0146 & G01571 & (Reed et al., 2000a) \\
\hline G. gallus & ADL0147 & G01572 & (Reed et al., 2000a) \\
\hline G. gallus & ADL0149 & G01574 & (Reed et al., 2000a) \\
\hline G. gallus & ADL0150 & G01575 & (Reed et al., 2000a) \\
\hline G. gallus & ADL0155 & G01742 & (Reed et al., 2000a) \\
\hline G. gallus & ADL0158 & G01582 & (Reed et al., 2000a) \\
\hline G. gallus & ADL0160 & G01584 & (Reed et al., 2000a) \\
\hline G. gallus & ADL0171 & G01593 & (Reed et al., 2000a) \\
\hline G. gallus & ADL0172 & G01594 & (Reed et al., 2000a) \\
\hline G. gallus & ADL0176 & G01598 & (Reed et al., 2000a) \\
\hline G. gallus & ADL0179 & G01601 & (Reed et al., 2000a) \\
\hline G. gallus & ADL0181 & G01603 & (Reed et al., 2000a) \\
\hline G. gallus & ADL0230 & G01650 & (Reed et al., 2000a) \\
\hline G. gallus & ADL0234 & G01654 & (Reed et al., 2000a) \\
\hline G. gallus & ADL0254 & G01674 & (Reed et al., 2000a) \\
\hline G. gallus & ADL0262 & G01682 & (Reed et al., 2000a) \\
\hline G. gallus & ADL0266 & G01686 & (Reed et al., 2000a) \\
\hline G. gallus & ADL0267 & G01687 & (Reed et al., 2000a) \\
\hline G. gallus & ADL0268 & G01688 & (Reed et al., 2000a) \\
\hline G. gallus & ADL0272 & G01692 & (Reed et al., 2000a) \\
\hline G. gallus & ADL0279 & G01699 & (Reed et al., 2000a) \\
\hline G. gallus & ADL0306 & G01721 & (Reed et al., 2000a) \\
\hline G. gallus & ADL0315 & G16117 & (Pang et al., 1999) \\
\hline G. gallus & ADL210 & G01630 & (Reed et al., 2000a) \\
\hline G. gallus & HUJ0005A & L10231 & (Reed et al., 2000a) \\
\hline G. gallus & HUJ0006A & L10294 & (Reed et al., 2000a) \\
\hline G. gallus & LEI0089 & X83239 & (Gibbs et al., 1997) \\
\hline G. gallus & LEI0098 & $\mathrm{X} 82860$ & (Gibbs et al., 1997) \\
\hline G. gallus & LEI0100 & X82859 & (Gibbs et al., 1997) \\
\hline G. gallus & LEI0101 & X82805 & (Gibbs et al., 1997) \\
\hline G. gallus & LEI0103 & X82796 & (Gibbs et al., 1997) \\
\hline G. gallus & LEI0106 & X82854 & (Gibbs et al., 1997) \\
\hline G. gallus & LEI0107 & X83253 & (Gibbs et al., 1997) \\
\hline G. gallus & LEI0108 & X85517 & (Gibbs et al., 1997) \\
\hline G. gallus & LEI0112 & X82789 & (Gibbs et al., 1997) \\
\hline
\end{tabular}


Continued.

\begin{tabular}{|c|c|c|c|}
\hline Origin species & Marker name & GenBank accession No. & Reference \\
\hline G. gallus & LEI0147 & X83256 & (Reed et al., 2000a) \\
\hline G. gallus & LEI126 & X82799 & (Reed et al., 2000a) \\
\hline G. gallus & LEI132 & X82856 & (Reed et al., 2000a) \\
\hline G. gallus & LEI161 & X85524 & (Reed et al., 2000a) \\
\hline G. gallus & LEI169 & X85535 & (Gibbs et al., 1997) \\
\hline G. gallus & MCW0005 & - & (Reed et al., 2000a) \\
\hline G. gallus & MCW0023 & - & (Reed et al., 2000a) \\
\hline G. gallus & MCW0080 & - & (Reed et al., 2000a) \\
\hline G. gallus & MCW0169 & - & (Reed et al., 2000a) \\
\hline G. gallus & MCW0228 & - & (Reed et al., 2000a) \\
\hline G. gallus & MCW0246 & - & (Reed et al., 2000a) \\
\hline G. gallus & MCW0300 & - & (Reed et al., 2000a) \\
\hline G. gallus & MCW0331 & - & (Reed et al., 2000a) \\
\hline G. gallus & MCW211 & G31988 & (Reed et al., 2000a) \\
\hline G. gallus & UMA1.019 & - & (Reed et al., 2000a) \\
\hline M. gallopavo & MNT001 & AF176506 & (Reed et al., 2000b) \\
\hline M. gallopavo & MNT003 & AF176508 & (Reed et al., 2000b) \\
\hline M. gallopavo & MNT005 & AF176510 & (Reed et al., 2000b) \\
\hline M. gallopavo & MNT006 & AF176511 & (Reed et al., 2000b) \\
\hline M. gallopavo & MNT007 & AF176512 & (Reed et al., 2000b) \\
\hline M. gallopavo & MNT011 & AF482370 & (Reed et al., 2002) \\
\hline M. gallopavo & MNT019 & AF482378 & (Reed et al., 2002) \\
\hline M. gallopavo & MNT020 & AF482379 & (Reed et al., 2002) \\
\hline M. gallopavo & MNT021 & AY235034 & (Reed et al., 2003) \\
\hline M. gallopavo & MNT041 & AY235054 & (Reed et al., 2003) \\
\hline M. gallopavo & MNT043 & AY235056 & (Reed et al., 2003) \\
\hline M. gallopavo & MNT044 & AY235057 & (Reed et al., 2003) \\
\hline M. gallopavo & MNT049 & AY235062 & (Reed et al., 2003) \\
\hline M. gallopavo & MNT058 & AY235071 & (Reed et al., 2003) \\
\hline M. gallopavo & MNT061 & AY235074 & (Reed et al., 2003) \\
\hline M. gallopavo & MNT118 & AF540421 & (Dranchak et al., 2003) \\
\hline M. gallopavo & MNT127 & AY235106 & (Reed et al., 2003) \\
\hline M. gallopavo & MNT129 & AF540431 & (Dranchak et al., 2003) \\
\hline M. gallopavo & MNT130 & AF540432 & (Dranchak et al., 2003) \\
\hline M. gallopavo & MNT131 & AY235107 & (Reed et al., 2003) \\
\hline M. gallopavo & MNT139 & AY235115 & (Reed et al., 2003) \\
\hline M. gallopavo & MNT141 & AF540433 & (Dranchak et al., 2003) \\
\hline M. gallopavo & MNT160 & AY235129 & (Reed et al., 2003) \\
\hline M. gallopavo & MNT171 & AY235139 & (Reed et al., 2003) \\
\hline M. gallopavo & MNT181 & AY235149 & (Reed et al., 2003) \\
\hline M. gallopavo & MNT188 & AY235156 & (Reed et al., 2003) \\
\hline M. gallopavo & MNT201 & AY235168 & (Reed et al., 2003) \\
\hline M. gallopavo & MNT204 & AY235171 & (Reed et al., 2003) \\
\hline M. gallopavo & MNT211 & AY235176 & (Reed et al., 2003) \\
\hline M. gallopavo & MNT215 & AY235180 & (Reed et al., 2003) \\
\hline M. gallopavo & MNT219 & AY235184 & (Reed et al., 2003) \\
\hline M. gallopavo & MNT220 & AY235185 & (Reed et al., 2003) \\
\hline M. gallopavo & MNT221 & AY235186 & (Reed et al., 2003) \\
\hline M. gallopavo & MNT222 & AY235187 & (Reed et al., 2003) \\
\hline M. gallopavo & MNT224 & AY235189 & (Reed et al., 2003) \\
\hline
\end{tabular}

Continued on next page 
Continued.

\begin{tabular}{|c|c|c|c|}
\hline Origin species & Marker name & GenBank accession No. & Reference \\
\hline M. gallopavo & MNT226 & AY235191 & (Reed et al., 2003) \\
\hline M. gallopavo & MNT249 & AY552821 & (Knutson et al., 2004) \\
\hline M. gallopavo & MNT250 & AY552822 & (Knutson et al., 2004) \\
\hline M. gallopavo & MNT275 & AY552847 & (Knutson et al., 2004) \\
\hline M. gallopavo & MNT276 & AY552848 & (Knutson et al., 2004) \\
\hline M. gallopavo & MNT291 & AY552863 & (Knutson et al., 2004) \\
\hline M. gallopavo & MNT399 & AY926537 & (Chaves et al., 2006) \\
\hline M. gallopavo & MNT400 & AY926538 & (Chaves et al., 2006) \\
\hline M. gallopavo & MNT401 & AY926539 & (Chaves et al., 2006) \\
\hline M. gallopavo & MNT402 & AL592658 & (Chaves et al., 2006) \\
\hline M. gallopavo & MNT405 & AY926540 & (Chaves et al., 2006) \\
\hline M. gallopavo & MNT407 & AY926542 & (Chaves et al., 2006) \\
\hline M. gallopavo & MNT409 & AL593356 & (Chaves et al., 2006) \\
\hline M. gallopavo & MNT410 & AL592718 & (Chaves et al., 2006) \\
\hline M. gallopavo & MNT411 & AY926544 & (Chaves et al., 2006) \\
\hline M. gallopavo & MNT414 & AY926546 & (Chaves et al., 2006) \\
\hline M. gallopavo & MNT416 & AY926548 & (Chaves et al., 2006) \\
\hline M. gallopavo & MNT427 & AL592640 & (Chaves et al., 2006) \\
\hline M. gallopavo & MNT429 & AL592644 & (Chaves et al., 2006) \\
\hline M. gallopavo & MNT433 & AL592677 & (Chaves et al., 2006) \\
\hline M. gallopavo & MNT436 & AL592829 & (Chaves et al., 2006) \\
\hline M. gallopavo & MNT437 & AL593814 & (Chaves et al., 2006) \\
\hline M. gallopavo & MNT442 & AL593052 & (Chaves et al., 2006) \\
\hline M. gallopavo & MNT443 & AL593060 & (Chaves et al., 2006) \\
\hline M. gallopavo & MNT448 & AL593178 & (Chaves et al., 2006) \\
\hline M. gallopavo & MNT450 & AL593308 & (Chaves et al., 2006) \\
\hline M. gallopavo & MNT453 & AL593364 & (Chaves et al., 2006) \\
\hline M. gallopavo & MNT454 & AL593401 & (Chaves et al., 2006) \\
\hline M. gallopavo & MNT456 & AL593466 & (Chaves et al., 2006) \\
\hline M. gallopavo & MNT458 & AL593487 & (Chaves et al., 2006) \\
\hline M. gallopavo & MNT466 & AL593563 & (Chaves et al., 2006) \\
\hline M. galopavo & MNT406 & AY926541 & (Chaves et al., 2006) \\
\hline
\end{tabular}

Origin species of the marker; Marker name; GenBank accession number; Article reference. In bold, specific but not polymorphic markers in grey partridge.

\section{REFERENCES (Supplementary material)}

Chaves LD, Knutson TP, Krueth SB and Reed KM (2006). Using the chicken genome sequence in the development and mapping of genetic markers in the turkey (Meleagris gallopavo). Anim. Genet. 37: 130-138.

Dranchak PK, Chaves LD, Rowe JA and Reed KM (2003). Turkey microsatellite loci from an embryonic cDNA library. Poult. Sci. 82: 526-531.

Gibbs M, Dawson DA, McCamley C, Wardle AF, et al. (1997). Chicken microsatellite markers isolated from libraries enriched for simple tandem repeats. Anim. Genet. 28: 401-417.

Knutson TP, Chaves LD, Hall MK and Reed KM (2004). One hundred fifty-four genetic markers for the turkey (Meleagris gallopavo). Genome 47: 1015-1028.

Pang SW, Ritland C, Carlson JE and Cheng KM (1999). Japanese quail microsatellite loci amplified with chicken-specific primers. Anim. Genet. 30: 195-199.

Reed KM, Mendoza KM and Beattie CW (2000a). Comparative analysis of microsatellite loci in chicken and turkey. Genome 43: 796-802. 
Reed KM, Roberts MC, Murtaugh J, Beattie CW, et al. (2000b). Eight new dinucleotide microsatellite loci in turkey (Meleagris gallopavo). Anim. Genet. 31: 140.

Reed KM, Chaves LD and Rowe JA (2002). Twelve new turkey microsatellite loci. Poult. Sci. 81: 1789-1791.

Reed KM, Chaves LD, Hall MK, Knutson TP, et al. (2003). Microsatellite loci for genetic mapping in the turkey (Meleagris gallopavo). Anim. Biotechnol. 14: 119-131. 